\title{
Gain and loss from transcatheter intra-arterial limb infusion of cisplatin for extremity osteosarcoma: a retrospective study of 99 cases in the past six years
}

This article was published in the following Dove Press journal: Cancer Management and Research

\author{
Lu Xie \\ Jie $X u^{\prime}$ \\ Sen Dong' \\ Jian $\mathrm{Gao}^{2}$ \\ Xiaodong Tang' \\ Taiqiang Yan' \\ Rongli Yang' \\ Wei Guo' \\ 'Musculoskeletal Tumor Center, Peking \\ University People's Hospital, Beijing, \\ People's Republic of China; \\ ${ }^{2}$ Catheterization Room \& Radiology \\ Department, Peking University People's \\ Hospital, Beijing, People's Republic of \\ China
}

Purpose: We intend to analyze the gain and loss from transcatheter intra-arterial (IA) limb infusion of cisplatin for extremity osteosarcoma in the past six years.

Patients and methods: Between December 2009 and August 2014, a total of 99 patients were analyzed for efficiency and followed up for long-term survival. Based on the different administration methods of cisplatin, we divided them into the following two cohorts: IA infusion of cisplatin $(n=48)$ and intravenous (IV) infusion of cisplatin $(n=51)$. Except for cisplatin, all the other drugs were given intravenously. Cisplatin was given intra-arterially with an infusion time of $3 \mathrm{hrs}$ or $6 \mathrm{hrs}$ using a pump, whereas historical controls received IV infusion of cisplatin within 60 mins. Tumor neovascularity (TNV) was analyzed before infusion, and subsequent arteriograms were compared with the baseline to determine percent changes. Definitive surgery with intended wide resection and postoperative pathological evaluation were performed in all these patients.

Results: No local or overall survival benefit was found in the patients preoperatively treated with IA infusion of cisplatin compared with IV infusion ( $P=0.336$ and 0.173 , respectively). Furthermore, serial arteriography was used to predict a good histologic response with an accuracy of $73.1 \%$ and a sensitivity of $100 \%$. There were sporadic cases with the telangiectatic subtype, which did not respond very well to IV chemotherapy, but later, the tumor obviously shrank after IA infusion of cisplatin. Our study also showed that the rates of the complication of skin and muscle necrosis were not so low as reported.

Conclusion: We did not observe any survival advantage of chemotherapy using IA infusion in osteosarcoma of the extremities. Arteriography for TNV can be used to predict the tumor histologic response. Malposition of the catheter might severely increase the complication of skin or muscle necrosis.

Keywords: intra-arterial chemotherapy, tumor neovascularity, telangiectatic osteosarcoma, complications

\section{Introduction}

According to the long-term follow-up of EURAMOS-1, aggressive use of neoadjuvant and adjuvant chemotherapy has significantly improved the five-year overall survival (OS) of osteosarcoma of extremities to 71\% (95\% CI: $68-73 \%) .{ }^{1}$ Response of the primary lesions to preoperative chemotherapy and surgical margins has emerged as important prognostic factors associated with metastasis. ${ }^{2}$ Although no major benefit was reported by some classic prospective trials investigating the advantage of intraarterial (IA) administration of systemic treatment for nonmetastatic extremity osteosarcoma, including IOR/OS-3, $,^{3,4} \mathrm{IOR} / \mathrm{OS}-5,{ }^{5}$ and COSS $86,{ }^{6}$ preoperative chemotherapy
Correspondence: Wei Guo Musculoskeletal Tumor Center, Peking University People's Hospital, No. II

Xizhimen South Street, Xicheng District, Beijing 100044, People's Republic of China

Tel +86 I 370 II 95504

Fax +861068318386

Email bonetumor@I63.com 
with IA administration of cisplatin was still used for decades to achieve a better tumor response by delivering chemotherapy directly into the arterial bed supplying the neoplasm as local extremity venous concentrations of a drug given via the IA route can reach up to five times higher than those obtained by using the systemic route of delivery for the same therapeutic agent. $^{7-10}$

In addition, early initiation of neoadjuvant chemotherapy may help prevent the development of micro-metastasis and reduce the likelihood of tumor dissemination during surgery. ${ }^{11}$ The histologic response to neoadjuvant therapy has been deemed one of the most important prognostic factors for long-term survival in nonmetastatic osteosarcoma. ${ }^{2}$ Adjustments to neoadjuvant therapy might entail either adding new agents like ifosfamide until the desired response was achieved or intensifying the dose or duration if the response was slow. ${ }^{12}$ Better local response induced by these methods might help surgeons resect all the pseudo-capsules, which were described by Picci et al, ${ }^{2}$ with highly collagenized fibrous tissue that was variable in width but continuous around the tumor mass to achieve better survival.

For osteosarcoma treated with chemotherapy, Response Evaluation Criteria in Solid Tumors (RECIST) ${ }^{13} 1.1$ is not applicable because bone lesions are typically located in irregularly shaped bones, are difficult to measure accurately, and usually respond more slowly to treatment than soft tissue lesions. Various studies have reported that tumor neovascularity (TNV) evaluated by serial arteriography $4,6,14,15$ is more sensitive and precise in detecting the tumor response, which could further allow treatment individualization to achieve the maximum response and limit therapy-related toxicity. In recent data, ${ }^{16-18}$ we found that telangiectatic osteosarcoma is a chemo-sensitive subtype with the same long-term outcome like the other subtypes. But because it is often histologically filled with blood and is separated by septa containing highly malignant cells, it might not manifest an equally good local response as the other subtypes during neoadjuvant chemotherapy, ${ }^{16,19}$ which makes the limb salvage procedure impossible to perform.

Based on all the findings mentioned above, we sought to retrospectively compare the patients treated with IA infusion of cisplatin during neoadjuvant chemotherapy for extremity osteosarcoma with those treated with IV administration. Specifically, we analyzed the results of this study on the basis of the following questions: (1) Does serial IA infusion of cisplatin improve the local tumor control rate of limb salvage surgery? (2) Does the addition of ifosfamide or increase in the number of agents in the neoadjuvant setting improve the histologic response? (3) Can serial arteriography be highly sensitive and accurate in predicting the responses? (4) Can IA infusion of cisplatin for telangiectatic osteosarcoma be beneficial for limb salvage? (5) What is the incidence of complication of IA administration of cisplatin in the real world and how can we avoid it?

\section{Patients and methods}

Between December 2009 and August 2014, 119 consecutive extremity osteosarcoma patients were initially treated in Peking University People's Hospital. Among them, 3 patients received the operation without adjuvant chemotherapy, 2 patients did not receive surgery because of tumor progression, and 15 patients were lost to follow-up. Finally, 99 patients were analyzed for local efficacy and long-term survival. Among them, 48 patients received IA infusion of cisplatin on more than one occasion, while 51 patients received only IV infusion of all systemic chemotherapeutic agents during the same period. There were 60 males $(60.6 \%)$ and 39 females $(39.4 \%)$ in the study. According to Collins et al's futher subclassification, there were 26 children $(26.3 \%), 34$ adolescents $(34.3 \%)$, and 39 adults $(39.4 \%) .{ }^{20}$ Tumors were located in the distal femur in 56 out of the 99 patients $(56.6 \%)$, in the proximal tibia in 25 out of the 99 patients $(25.3 \%)$, in the proximal femur in 2 out of the 99 patients $(2.0 \%)$, in the proximal humerus in 12 out of the 99 patients $(12.1 \%)$, in the scapula in 2 out of the 99 patients $(2.0 \%)$, in the distal tibia and fibula in 1 out of the 99 patients $(1.0 \%)$, and on the palm in 1 out of the 99 patients $(1.0 \%)$. A total of 78 patients were initially found to have localized osteosarcoma, while 21 patients manifested a metastatic pattern during diagnosis. Among them, 17 out of the 99 patients (17.2\%) had the chondroblastic subtype, 73 out of the 99 patients (73.7\%) had the osteoblastic or fibroblastic or mixed subtype, 5 out of the 99 patients $(5.1 \%)$ had telangiectatic osteosarcoma, 3 out of the 99 patients $(3.0 \%)$ had the small cell type, and 1 patient had high-grade surface type osteosarcoma (see Table 1). The median follow-up time for patients treated with IA infusion of cisplatin was 62.6 months (range, 13.286.3 months), and the median follow-up time for those treated with IV infusion of cisplatin was 51.1 months (range, 7.1-80.1 months).

We generally recommended neoadjuvant therapy followed by delayed definitive surgery and adjuvant chemotherapy after diagnosis of high-grade osteosarcoma. Patients routinely received neoadjuvant chemotherapy according to the Peking University People's Hospital-Osteosarcoma 
Table I Patient demographics

\begin{tabular}{|c|c|c|c|c|c|c|}
\hline \multirow[t]{2}{*}{ Characteristic } & \multicolumn{2}{|c|}{ No. of patients } & \multicolumn{2}{|c|}{ For EFS } & \multicolumn{2}{|c|}{ For OS } \\
\hline & IV infusion & IA infusion & $P_{u}{ }^{a}$ & $P_{m}^{b}$ & $P_{u}$ & $P_{m}$ \\
\hline Total & 51 & 48 & 0.233 & $N / A$ & 0.176 & $N / A$ \\
\hline $\begin{array}{l}\text { Gender } \\
\text { Male } \\
\text { Female }\end{array}$ & $\begin{array}{l}26(51.0 \%) \\
25(49.0 \%)\end{array}$ & $\begin{array}{l}34(70.8 \%) \\
14(29.2 \%)\end{array}$ & 0.057 & $N / A$ & 0.133 & N/A \\
\hline $\begin{array}{l}\text { Age }^{c} \\
\text { Child } \\
\text { Adolescent } \\
\text { Adult }\end{array}$ & $\begin{array}{l}16(31.4 \%) \\
16(31.4 \%) \\
19(37.2 \%)\end{array}$ & $\begin{array}{l}10(20.8 \%) \\
18(37.5 \%) \\
20(41.7 \%)\end{array}$ & 0.349 & N/A & 0.145 & N/A \\
\hline $\begin{array}{l}\text { Pathological subtypes } \\
\text { Chondroblastic } \\
\text { Common type without chondroblastic } \\
\text { Telangiectatic type } \\
\text { Small cell type } \\
\text { High-grade surface type }\end{array}$ & $\begin{array}{l}7(13.7 \%) \\
37(72.5 \%) \\
4(7.8 \%) \\
2(3.9 \%) \\
1(2.0 \%)\end{array}$ & $\begin{array}{l}10(20.8 \%) \\
36(75.0 \%) \\
1(2.1 \%) \\
1(2.1 \%) \\
0(0 \%)\end{array}$ & 0.395 & $N / A$ & 0.564 & N/A \\
\hline $\begin{array}{l}\text { Locations } \\
\text { Distal femur } \\
\text { Proximal tibia } \\
\text { Proximal femur } \\
\text { Proximal humerus } \\
\text { Others }\end{array}$ & $\begin{array}{l}23(45.1 \%) \\
10(19.6 \%) \\
2(3.9 \%) \\
12(23.6 \%) \\
4(7.8 \%)\end{array}$ & $\begin{array}{l}33(68.8 \%) \\
15(31.2 \%) \\
0(0 \%) \\
0(0 \%) \\
0(0 \%)\end{array}$ & 0.412 & $N / A$ & 0.951 & N/A \\
\hline $\begin{array}{l}\text { Stage (Ennecking) } \\
\text { IIB } \\
\text { III }\end{array}$ & $\begin{array}{l}35(68.6 \%) \\
16(31.4 \%)\end{array}$ & $\begin{array}{l}43(89.6 \%) \\
5(10.4 \%)\end{array}$ & 0.003 & 0.003 & 0.000 & 0.000 \\
\hline $\begin{array}{l}\text { Surgical procedures } \\
\text { Limb salvage } \\
\text { Amputation }\end{array}$ & $\begin{array}{l}49(96.1 \%) \\
2(3.9 \%)\end{array}$ & $\begin{array}{l}48(100.0 \%) \\
0(0 \%)\end{array}$ & 0.223 & N/A & 0.692 & N/A \\
\hline $\begin{array}{l}\text { Surgical margins } \\
\text { RO } \\
\text { RI } \\
\text { N/A }\end{array}$ & $\begin{array}{l}37(72.5 \%) \\
9(17.6 \%) \\
5(9.8 \%)\end{array}$ & $\begin{array}{l}43(89.6 \%) \\
5(10.4 \%) \\
0(0 \%)\end{array}$ & 0.023 & 0.135 & 0.024 & 0.283 \\
\hline $\begin{array}{l}\text { Preoperative chemotherapy time interval } \\
\text { Less than } 8 \text { weeks } \\
\text { More than } 8 \text { weeks }\end{array}$ & $\begin{array}{l}21(41.2 \%) \\
30(58.8 \%)\end{array}$ & $\begin{array}{l}10(20.8 \%) \\
38(79.2 \%)\end{array}$ & 0.272 & N/A & 0.244 & N/A \\
\hline $\begin{array}{l}\text { Preoperative chemotherapy protocol } \\
\text { Without IFO } \\
\text { With IFO }\end{array}$ & $\begin{array}{l}\text { I3 (25.5\%) } \\
38(74.5 \%)\end{array}$ & $\begin{array}{l}10(20.8 \%) \\
38(79.2 \%)\end{array}$ & 0.846 & N/A & 0.931 & N/A \\
\hline $\begin{array}{l}\text { Perfusion time } \\
3 \mathrm{hrs} \\
6 \mathrm{hrs}\end{array}$ & $\begin{array}{l}0(0 \%) \\
0(0 \%)\end{array}$ & $\begin{array}{l}19(39.6 \%) \\
29(60.4 \%)\end{array}$ & 0.795 & N/A & 0.454 & N/A \\
\hline $\begin{array}{l}\text { Histological response } \\
\text { Good }^{\text {e }} \\
\text { Poor }\end{array}$ & $\begin{array}{l}20(39.2 \%) \\
31(60.8 \%)\end{array}$ & $\begin{array}{l}26(54.2 \%) \\
22(45.8 \%)\end{array}$ & 0.041 & 0.153 & 0.034 & 0.037 \\
\hline
\end{tabular}

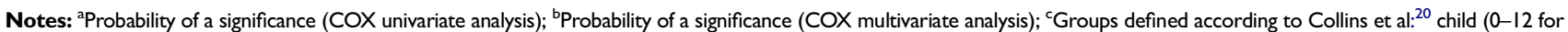
males and 0-1 I for females), adolescent ( $13-17$ for males and I $2-16$ for females), and adult ( $\geq 18$ for males and $\geq 17$ for females); ${ }^{\mathrm{d} A c c o r d i n g}$ to Enneking et al; ${ }^{24} \mathrm{e} U$ pon histopathological examination, the tumor response was assessed on the basis of the presence and extent of necrosis, which was assessed by a combination of gross and microscopic observations. Tumor necrosis was graded as Picci et al, ${ }^{23}$ tumor histopathological response grading system (Huvos classification), where we defined an equal to or more than $90 \%$ tumor necrosis rate as a good histological response and less than $90 \%$ as a poor response.

Abbreviations: EFS, event-free survival; IA, intra-arterial; IFO, Ifosfamide; IV, intravenous; N/A, not available; OS, overall survival. 
(PKUPH-OS) regimen, ${ }^{21}$ which began in 2008 (Figure 1). From our past experience, we usually selected those with relative tumor volume large ( $\geq 1 / 3$ of the involved bone) and those with $\geq 20 \%$ chondroid ground substance in the biopsy specimen to conduct IA infusion of cisplatin. Generally speaking, we divided neoadjuvant chemotherapy protocols with IA infusion of cisplatin into three small cohorts, and all of these protocols consisted of adriamycin/doxorubicin, cisplatin, and high-dose methotrexate with or without ifosfamide with a time interval of 6-9 weeks. Except for cisplatin, all the other drugs were given intravenously. Doxorubicin was usually given for two courses preoperatively, and each course was given as a 3 -hr pump drip of $2 \times 30 \mathrm{mg} / \mathrm{m}^{2} / \mathrm{d}$ on consecutive days (weeks 0,6 ) after cisplatin infusions (weeks $0,6)$, followed by two courses of high-dose methotrexate at 8-12 g/m $\mathrm{m}^{2}$ with leucovorin rescue, $12 \times 9 \mathrm{mg} / \mathrm{m}^{2} / \mathrm{i} . \mathrm{m}$. per course. Ifosfamide at $5 \times 2.4 \mathrm{~g} / \mathrm{m}^{2}$ as $2-\mathrm{hr}$ infusions and uromitexan uroprotection was occasionally added to the protocol due to a slow response or huge tumor volume. Cisplatin was administered intravenously or intra-arterially after adequate IV hydration. For IA infusion, cisplatin was given via insertion of a catheter percutaneously by using the Seldinger technique $^{22}$ through the brachial or femoral artery under anesthesia and usually at $120 \mathrm{mg} / \mathrm{m}^{2}$ as a 3-hr/6-hr continuous infusion; while for IV administration, it was given at $100-120 \mathrm{mg} / \mathrm{m}^{2}$ as $6-\mathrm{hr}$ infusions.

Arteriograms were obtained before the administration of each dose of cisplatin. The last anteroposterior and lateral images obtained in the arteriographic sequence, which demonstrated complete contrast agent filling of the target trunk vessel (ie, the last full column), were chosen from each arteriogram used for interpretation and comparison with future studies. Only those cases with at least two IA infusions of cisplatin could evaluate the TNV change in tumors. The volume and intensity of TNV on the baseline arteriogram were assessed and compared with those on subsequent arteriograms to assess the rate of change of TNV as an indicator of the tumor response, and only those with more than $90 \%$ disappearance of TNV were considered a good response for TNV, or else they were considered a poor response for TNV.

Definitive surgery was scheduled at least 2 weeks after the end of routine cisplatin infusion with the intention of achieving wide surgical margins. All pathology slides were reviewed by two senior pathologists (SKK and SDH). They evaluated all surgical specimens and were blinded to the clinical status. The surgical margins were identified retrospectively by two separate senior surgeons according to the preoperative MRI of the tumors as well as two separate pathologists' gross and microscopic evaluations. If they have diverse opinions on these cases, a third surgeon and pathologist would be asked to identify. Upon histopathological examination, the tumor response was assessed on the basis of the presence and extent of necrosis, which was determined by a combination of gross and microscopic observations. ${ }^{23}$ Tumor necrosis was graded as per Picci et $\mathrm{al}^{23}$ histopathological response grading system (Huvos classification), where at least 90\% necrosis was defined as a good response and less than $90 \%$ necrosis was defined as a

Protocol for osteosarcoma in Peking University Poeple's Hospital

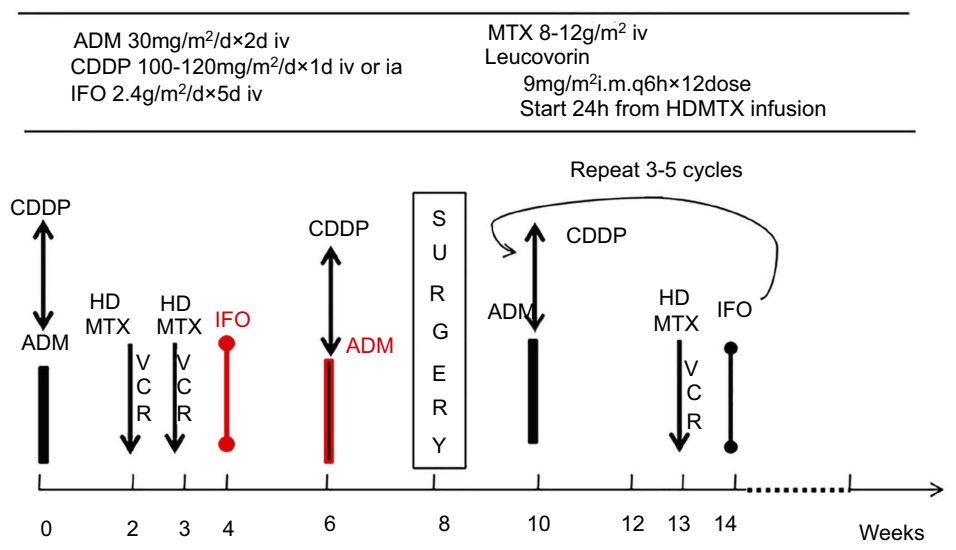

A: APMMAP: Without ifosfamide at W4,duration of preoperative chemotherapy of almost 6 wks;

B: APMMAP: Without adriamycin at W6,duration of preoperative chemotherapy of almost 7 wks;

C: APMMAP: Like the protocol upwards, duration of preoperative chemotherapy of almost 9 wks.

Figure I The chemo-regimen for osteosarcoma patients in musculoskeletal tumor center of Peking University People's Hospital (Peking University People's HospitalOsteosarcoma, PKUPH-OS). 
poor response. Adjuvant chemotherapy was sequentially performed with the PKUPH-OS protocol (Figure 1) for at least 30 weeks after surgery.

Baseline chest CT, bone scans, or positron emission tomography (PET)/CT was used to assess metastatic disease. Patients' follow-up included chest CT, local radiographs, and ultrasound/local CT scans every 3 months, as well as bone scans or PET/CT every 6 months during treatment and two years after completion of adjuvant therapy. During the third to fifth year, chest and pelvic CTs were obtained every 6 months and then yearly thereafter.

Descriptive statistics were used to display demographic data. Kaplan-Meier analysis was used to determine OS, event-free survival (EFS), and recurrence-free survival. We calculated the tumor response rates in different groups as the percentage of patients who had a tumor necrosis rate of more than $90 \%,{ }^{3,6,10,12}$ which, according to the results of EURAMOUS-1, ${ }^{1}$ should be $51.9 \%$ (724/1395) for routine chemo-protocols. If we want tumor response elevated from $51.9 \%$ to $70 \%$ with setting of $\alpha$-error at 0.05 and $\beta$-error at 0.20 , at least 61 patients were needed for statistical calculation. We calculated EFS from the date of diagnosis to the last follow-up, local recurrence, distant metastasis, or death. We defined recurrence-free survival from the time of surgery to the date of local recurrence. We compared all the clinical pathological characteristics by Cox univariate analysis of EFS and OS. We also performed subsequent Cox proportional hazards analysis of variables to identify factors associated with survival. Correlation was made between TNV decrease of at least $90 \%$ by arteriography and at least $90 \%$ tumor necrosis by histologic examination. A $P$-value $<0.05$ was considered significant. Analysis was performed using the SPSS software package (SPSS Inc., Chicago, IL, USA).

Ethical approval for the study was provided by the ethics committee of Peking University People's Hospital. All data used in the study were obtained from the existing medical records of our hospital, and the requirement for obtaining written informed consent was waived. All patient data were treated with confidentiality, in accordance with the Declaration of Helsinki.

\section{Results}

Does serial intra-arterial infusion of cisplatin improve the local tumor control rate of limb salvage surgery?

Overall, 78 IA cisplatin infusions were given to 48 patients (26 evaluable for TNV with repeated arteriograms) with adequate documentation of the respective procedures. Local anesthesia and sedation were reported in 45 out of these 48 patients, while general anesthesia and sedation were used preferentially in three younger children. The infusion time was reported to be the scheduled $3 \mathrm{hrs}$ in 19 out of these 48 patients $(39.6 \%)$, and it reached or exceeded the scheduled 6 hrs in 29 out of these 48 patients $(60.4 \%)$.

With a median follow-up time of 58.3 months (IQR 25.4, 91.2), 97 out of the 99 patients $(98.0 \%)$ initially received limb-sparing surgeries, while 2 out of the 99 patients $(2.0 \%)$ received amputation directly. The limb salvage rate was $100 \%(48 / 48)$ in the IA group and 96\% (49/51) in the IV group. After reviewing all these specimens and imagings, $72.5 \%(37 / 51)$ of the patients in IV group had $\mathrm{R} 0$ resection, $17.6 \%(9 / 51)$ had R1 resection while $9.8 \%(5 / 51)$ could not be evaluated retrospectively. In the IA group, 89.6\% (43/48) had $\mathrm{R} 0$ resection and $10.4 \%(5 / 48)$ had $\mathrm{R} 1$ resection. Until the last follow-up, 50 out of the 99 patients $(50.5 \%)$ were alive without any evidence of disease, 7 out of the 99 patients $(7.1 \%)$ were alive with the disease, and 42 out of the 99 patients (42.4\%) had died of the disease. The local-recurrence rate was $16.7 \%(8 / 48)$ in the IA group and $23.5 \%(12 / 51)$ in the IV group without any obvious statistical difference $(P=0.336)$ (Figure 2). Nevertheless, there was a slight trend toward improvement of local tumor control in the early phase (from 12 to 36 months after surgery). The total tumor response rate was $54.2 \%(26 / 48)$ in the IA group and $39.2 \%(20 / 51)$ in the IV group. For long-term survival, the five-year EFS rates were $60.4 \%(95 \%$ CI $46.5 \%, 74.3 \%)$ in the IA group and $50.1 \%(95 \%$ CI $36.2 \%, 64.0 \%)$ in the IV group without any obvious statistical differences $(P=0.158)$. Besides, the respective five-year OS rates were 64.1\% (95\% CI 50.4\%, 77.82\%) in the IA group and $55.9 \%(95 \%$ CI $42.0 \%, 69.8 \%)$ in the IV group without any statistical differences $(P=0.173)$.

After controlling for confounders, such as gender, age, pathological subtypes, locations of primary tumors, stage, $^{24}$ and surgical type and surgical margins, only stage II (HR, 0.322; 95\% CI, 0.170-0.612; $P=0.001$ ) and good histological response (HR, 0.502; 95\% CI, 0.263-0.958; $P=0.037$ ) remained independent factors associated with OS (Figures 3 and 4).

\section{Does the addition of ifosfamide or increase} in the number of agents in the neoadjuvant setting improve the histologic response?

Due to retrospective data and their irregularity, we divided the neoadjuvant chemotherapy protocols into several cohorts 


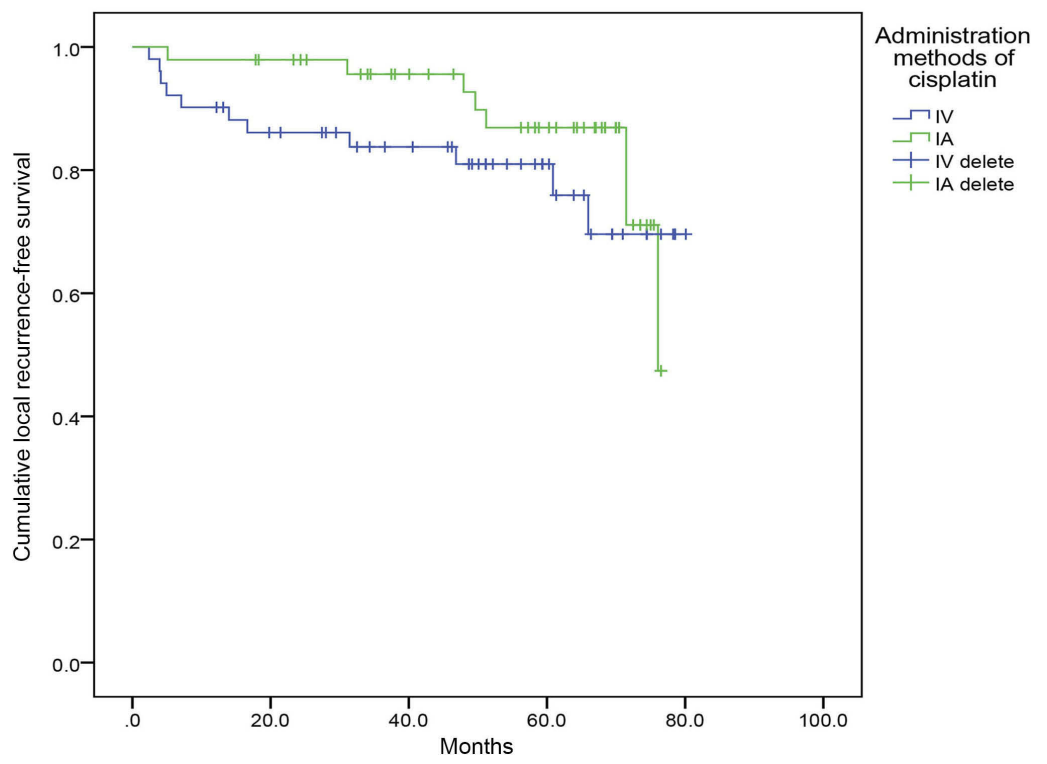

Figure 2 The Kaplan-Meier survival curve for local-recurrence free survival for IA (intra-arterial) and IV (intravenous) infusion of cisplatin extremity osteosarcoma patients initially treated in Peking University People's Hospital.

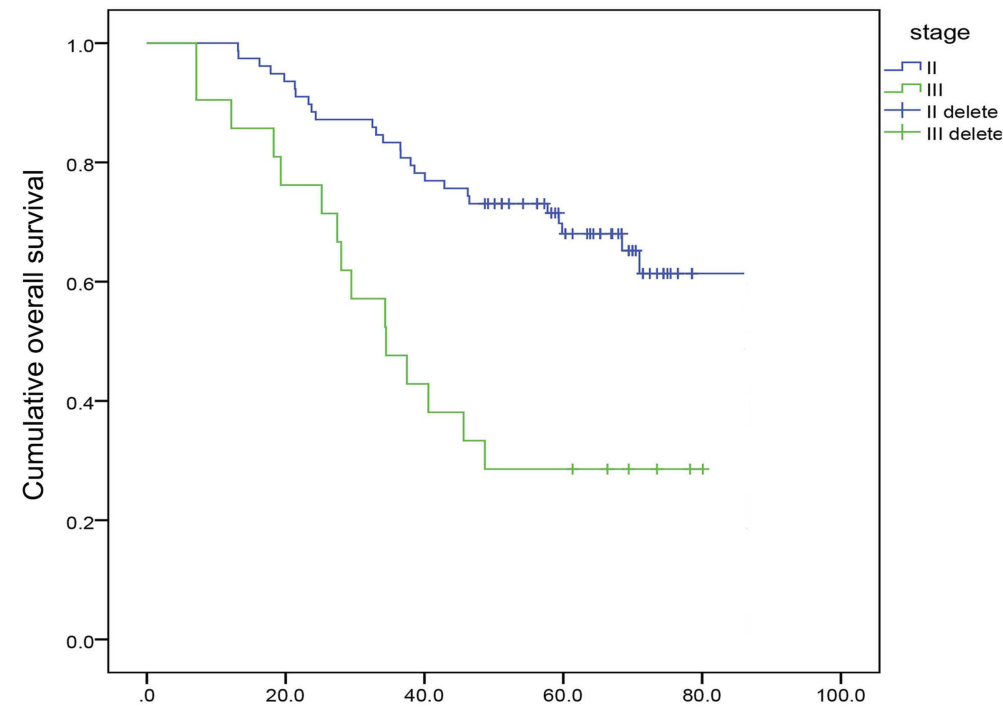

Figure 3 The Kaplan-Meier survival curve for overall survival for nonmetastatic and metastatic extremity osteosarcoma patients initially treated in Peking University People's Hospital.

to check whether the addition of ifosfamide or increase in the number of chemotherapeutic agents is helpful to improve tumor responses or survival. All the protocols using IA infusion are summarized in Table 2. A total of 10 out of the 48 patients received the APMMAP protocol with the duration of neoadjuvant chemotherapy of approximately 6 weeks. The percentage of patients with $\geq 90 \%$ tumor necrosis rate was $30 \%$. During the same period, 26 out of the 48 patients received the APMMIP protocol with the duration of neoadjuvant chemotherapy of almost 7 weeks, while 21 out of the
48 patients received the APMMIAP protocol with 9 weeks of neoadjuvant chemotherapy, inducing tumor response rates of $50 \%$ and $66.7 \%$, respectively. We could observe a trend for the elevation of tumor necrosis rates after the addition of ifosfamide or increase in the number of chemotherapeutic agents. However, on taking together with those receiving IV chemotherapy, we divided the population into those treated with ifosfamide $(\mathrm{N}=76)$ and those treated without ifosfamide $(\mathrm{N}=23)$ during neoadjuvant chemotherapy, and we found no obvious difference in EFS or OS $(P=0.846$ and 0.931, 


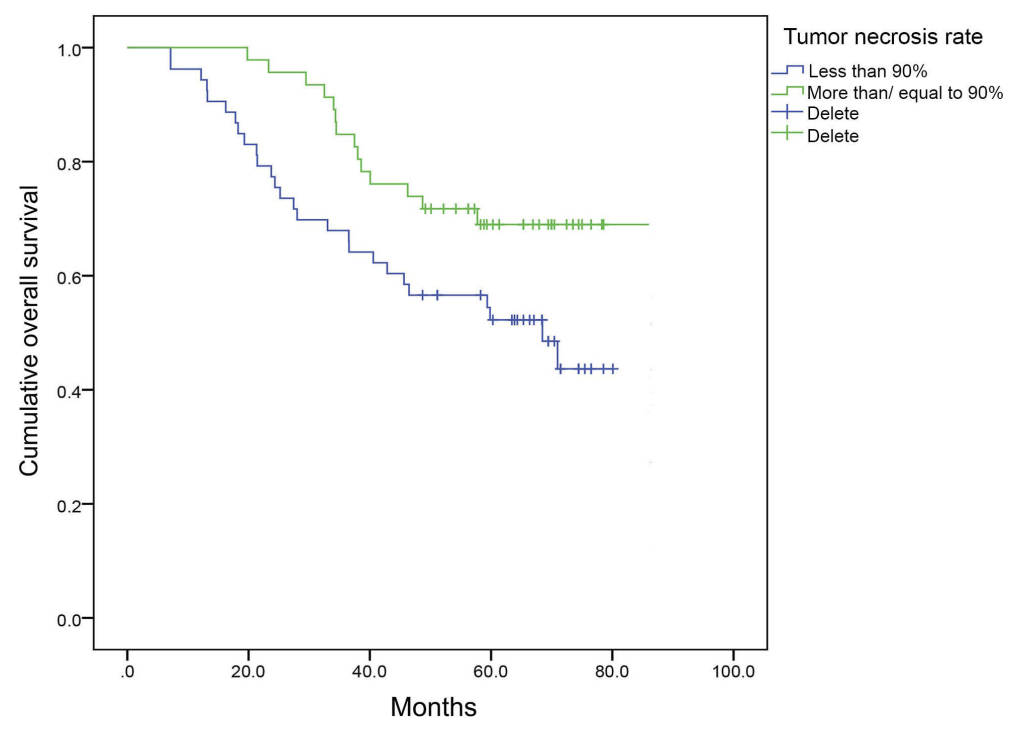

Figure 4 The Kaplan-Meier survival curve for overall survival for pathological good and poor response patients with osteosarcoma of extremities.

respectively, Table 1). To analyze the benefit of increasing the number of agents during neoadjuvant chemotherapy, we divided all the patients into those with a time interval of more than 8 weeks $(\mathrm{N}=68)$ and those with a time interval of less than 8 weeks $(\mathrm{N}=31)$, and we did not find any advantage in EFS or OS ( $P=0.272$ and 0.244 , respectively).

\section{Can serial arteriography be highly sensitive and accurate in predicting the responses?}

A total of 26 out of the 48 patients $(54.2 \%)$ received at least two IA infusions of cisplatin. The changes in TNV seen on angiograms after successive courses of chemotherapy were assessed. A good angiographic response was defined as $\geq 90 \%$ disappearance of TNV and tumor stain (Figure 5) or a total disappearance of tumor vascularity with slight persistence of the capillary stain. A poor response was defined as persistence or even more concentration of TNV and tumor stain (Figure 6). As shown in Table 3, TNV was decreased by at least $90 \%$ in $69.2 \%$ of the patients $(18 / 26)$ and by less than $90 \%$ in $30.8 \%$ of the patients $(8 / 26)$. For those who did not exhibit a good arteriographic response, we did not add more cycles of IA infusion as described in the literature. ${ }^{14}$ A good histologic response, defined as at least $90 \%$ tumor necrosis, was achieved in $42.3 \%$ of the tumors $(11 / 26)$. The arteriogram correctly predicted a good histologic response in 11 out of 11 patients, yielding a sensitivity of $100 \%$. In addition, the arteriogram correctly predicted a poor histologic response in 8 out of 15 patients, providing a specificity of $53.3 \%$. The overall accuracy in predicting the histologic response by evaluating the decrease in TNV on serial arteriograms was $73.1 \%$ (19 out of 26 patients).

\section{Can IA infusion of cisplatin for telangiectatic osteosarcoma be beneficial for limb salvage?}

There were five localized telangiectatic osteosarcoma patients in our study. Among them, four patients received IV infusion of systemic chemotherapy and one patient initially received IV infusion and then one session of IA administration of cisplatin because of local progression upon chemotherapy. The tumor in this patient clinically shrank afterward with a tumor necrosis rate of $95 \%$ after resection. The four patients treated with IV infusion of cisplatin showed a pathological tumor necrosis rate of $33 \%, 100 \%, 70.9 \%$, and $55.6 \%$. Because of the small sample size, we could not calculate any statistical arithmetic for survival. However, until the last follow-up, only the patient with a necrosis rate of $55.6 \%$, who received amputation as definitive surgery, developed pulmonary metastasis within 6 months after completion of adjuvant chemotherapy and died one year later. All the other four patients were alive without any evidence of disease.

\section{What is the incidence of complication of IA administration of cisplatin in the real world?}

Toxicity from doxorubicin, high-dose methotrexate, and ifosfamide were not found to be different from that stated 


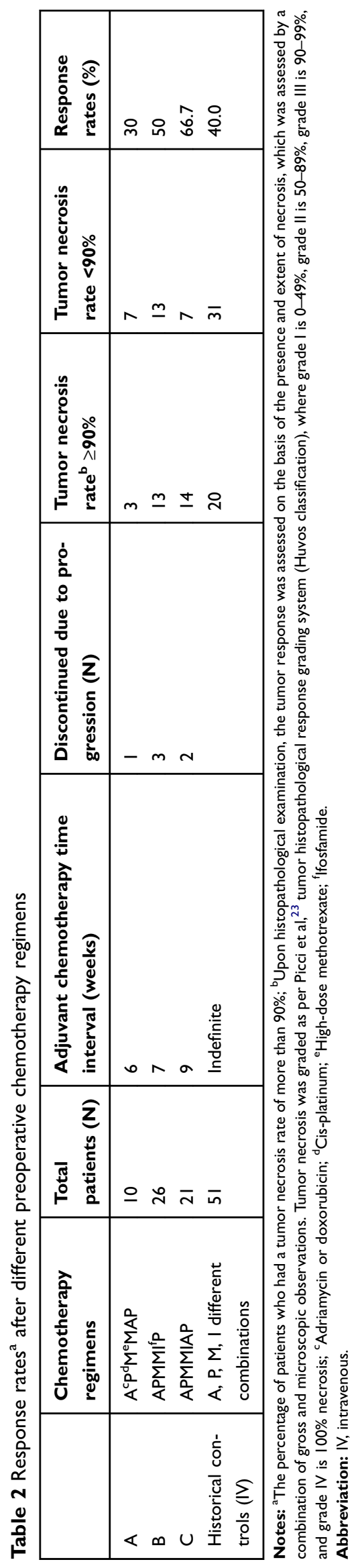

in previous reports. ${ }^{1,3-5,21}$ After 78 cycles of IA infusion of cisplatin, 13 out of the 48 patients treated with IA cisplatin developed painful local swelling followed by skin necrosis (Figure 7), muscle necrosis (Figure 8), or even bone necrosis. This usually occurred during the first cycle of chemotherapy. In all of these cases, the lesions healed in 14-20 days with discolored scars and did not affect the scheduled surgery.

We summarized the percentage of reported toxicities/ complications for cisplatin IA limb infusion according to the Common Terminology Criteria for Adverse Events (CTCAE) version 4.03. ${ }^{25,26}$ (Table 4) Another 13 out of the 48 patients complained of extremity pain without any reason. Furthermore, 2 out of the 48 patients experienced pathological fractures after IA treatment due to the dose intensity from the outset of treatment. The ototoxicity was not obvious in this study, which can be partly attributed to only two courses of cisplatin infusion at limited doses. The most common complications after IA chemotherapy, including nausea and vomiting, mucositis, neutropenia, and myelosuppression, are usually correlated with the toxicity of the agents themselves. We did not observe any hematoma at the puncture site or deep vein thrombosis in the extremity that received infusion, which might also be related to the procedure.

\section{Discussion}

The value of serial arteriography to assess tumor response, predict necrosis, and individualize the duration of a combined IV and IA neoadjuvant chemotherapy protocol in patients with high-grade osteosarcoma has been investigated for decades through retrospective $e^{7,9,27}$ or prospective studies. ${ }^{4,6,12}$ Although we are aware of the predictive accuracy of the change in TNV during serial arteriography to evaluate the local response of the tumor instead of morphological change of primary osteogenic tumors, ${ }^{3,10,13,14}$ this procedure has seldom been used recently because of the proven evidence of no long-term survival advantage. ${ }^{3,5,6,10,12,15,27}$ Nevertheless, it is believed that the introduction of aggressive chemotherapy has dramatically improved the prognosis of osteosarcoma, yielding a five-year OS rate in the range of $60-70 \%$ as compared with the previous approximate rate of $15-20 \%$ with only local treatment. ${ }^{1,2,7,12}$ The use of the IA route of administration of cisplatin allows an increase in the dose of the drug delivered to the tumor by as much as fivefold compared with the IV route without increasing the systemic concentration, effect, or toxicity. ${ }^{28,29}$ Especially for the patients with a localized lesion, early intensive intervention might be helpful not only for 

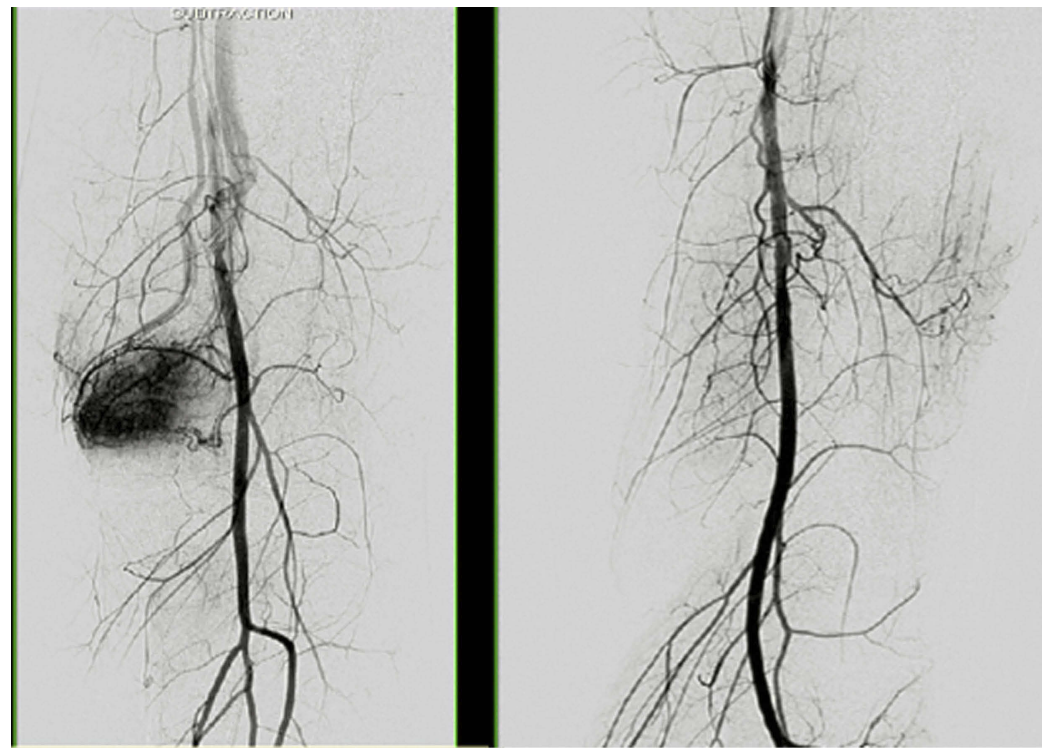

Figure 5 The angiogram showed a good angiographic response, which indicated as $\geq 90 \%$ disappearance of tumor vascularity and tumor stain.
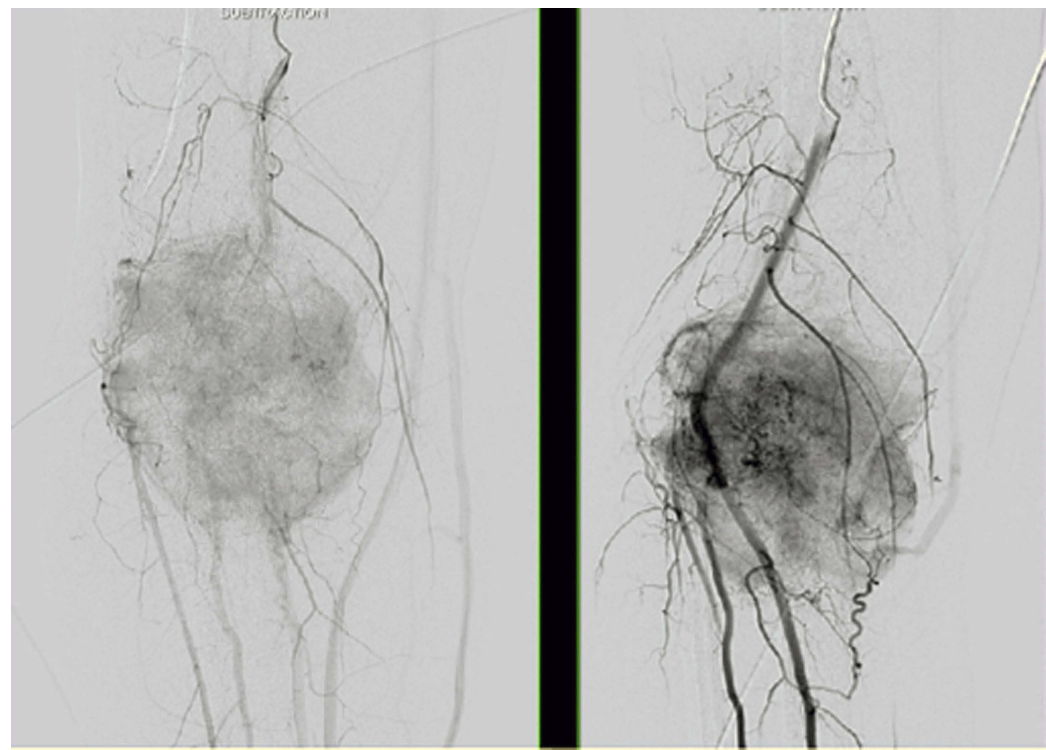

Figure 6 The angiogram showed a poor angiographic response, which indicated as persistence or even more concentration of tumor vascularity and tumor stain.

Table 3 Correlation of angiographic and histologic evaluation during neoadjuvant chemotherapy for osteosarcoma

\begin{tabular}{|l|l|l|l|}
\hline \multirow{2}{*}{ Angiographic assessment } & \multicolumn{2}{|l|}{ Histological assessment } & \multicolumn{2}{|l|}{} \\
\cline { 2 - 4 } & Good response & Poor response & Total \\
\hline Good response & 11 & 7 & 18 \\
Poor response & 0 & 8 & 8 \\
Total & 11 & 15 & 26 \\
\hline
\end{tabular}

Notes: Sensitivity $=100 \%$; Specificity $=53.3 \%$; and Accuracy $=73.1 \%$. 


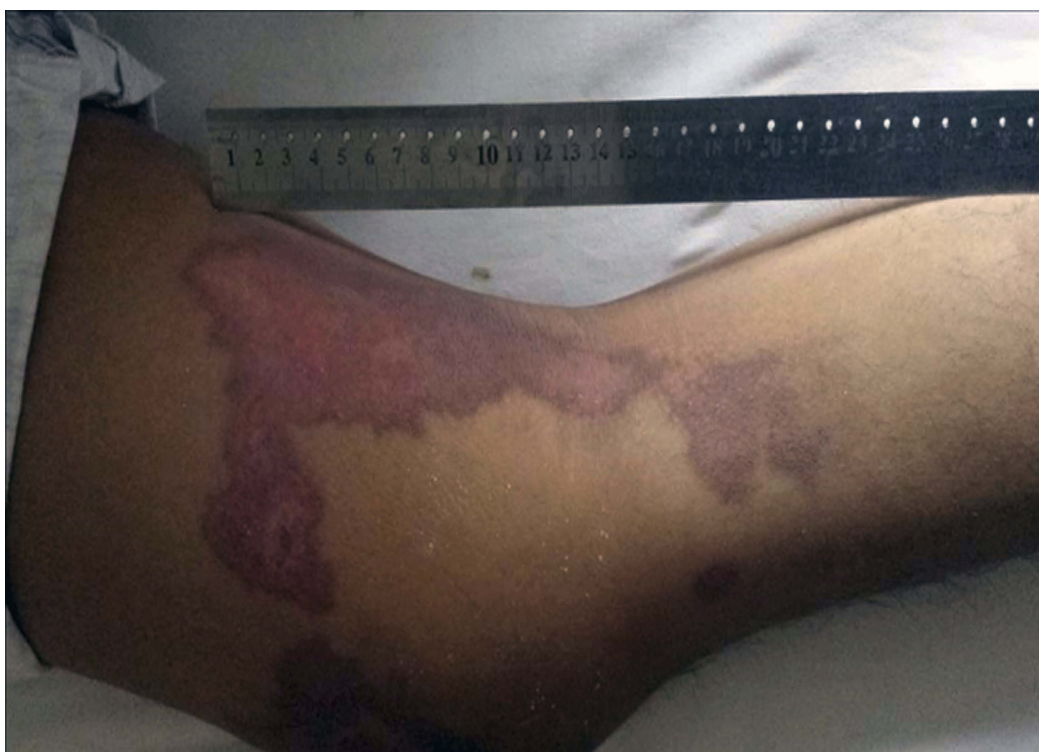

Figure 7 Skin necrosis after intra-arterial infusion of cisplatin.

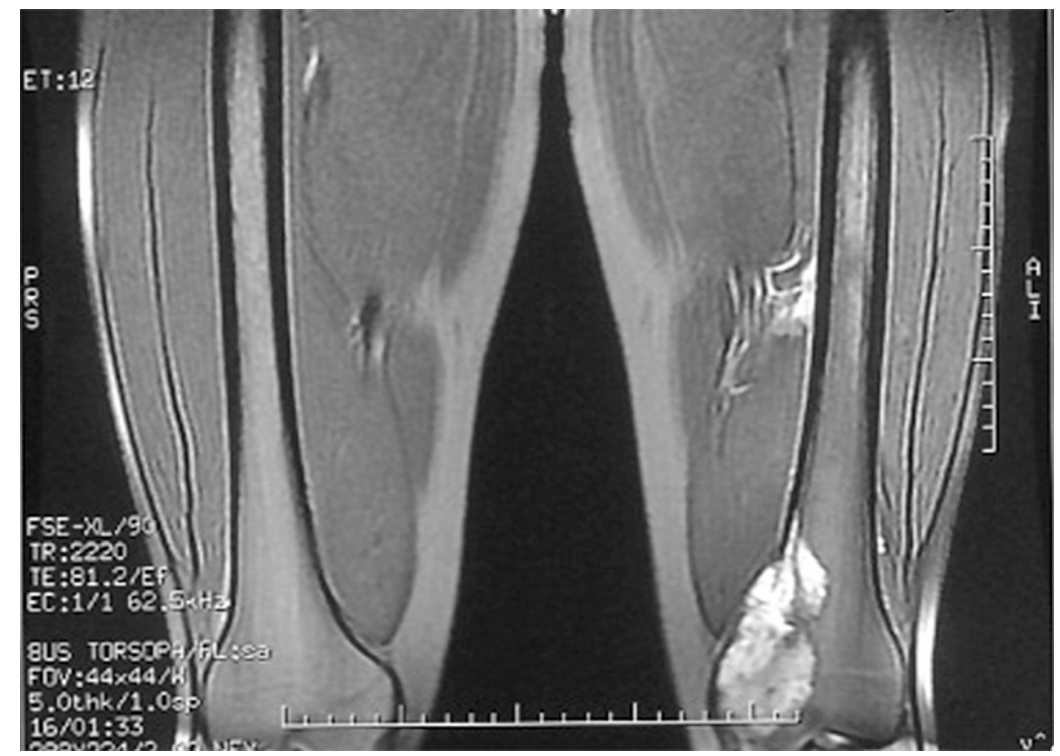

Figure 8 The magnetic resonance imaging manifestation of muscle necrosis after intra-arterial infusion of cisplatin.

facilitating limb salvage procedures but also in reducing local failure rates and preventing potential metastasis. We summarized our experience with IA infusion of cisplatin in retrospective cohorts to analyze the real gain and loss in this relatively uneconomical, uncomfortable, and complicated procedure.

In comparison with histologic controls treated with IV infusion of cisplatin, we did not find any statistical survival benefit for IA infusion; however, an improved local response rate was noted during IA neoadjuvant chemotherapy, and it tended to increase with addition of more number of neoadjuvant chemotherapeutic agents, which should be deemed to be in accordance with $\operatorname{COSS} 86^{12}$ showing the same local response rates by comparing multidrug systemic treatment including ifosfamide to IA infusion of cisplatin.

The histologic response to neoadjuvant therapy is one of the most important prognostic factors for long-term survival, which has been verified again in our study. At the same time, good or poor histologic responses could be precisely predicted by serial arteriography in our study with an accuracy of $73.1 \%$ and a sensitivity of $100 \%$. However, in patients who could not achieve disappearance of TNV to more than $90 \%$ after second IA infusion, we did 
Table 4 Percentage of reported toxicities/complications for CDDP intra-arterial limb infusion according to CTCAE version 4.03

\begin{tabular}{|l|l|l|l|l|}
\hline Adverse event & No. of courses & \multicolumn{3}{l|}{ Percentage of patients } \\
\cline { 3 - 5 } & & Grades 3-4 & $\mathbf{3} \mathbf{h r s}^{\mathbf{a}}$ & $\mathbf{6} \mathbf{h r s}^{\mathbf{b}}$ \\
\hline Skin ulceration (skin necrosis) & $10 / 78$ & $14.5 \%(7 / 48)$ & $10.5 \%(2 / 19)$ & $20.1 \%(6 / 29)$ \\
Soft tissue necrosis lower limb (muscle necrosis) & $5 / 78$ & $6.3 \%(3 / 48)$ & $5.3 \%(I / 19)$ & $10.3 \%(3 / 29)$ \\
Avascular necrosis (bone necrosis) & $2 / 78$ & $2.1 \%(1 / 48)$ & $0 \%(0 / 19)$ & $3.5 \%(1 / 29)$ \\
Pain in extremity & $23 / 78$ & $6.3 \%(3 / 48)$ & $26.3 \%(5 / 19)$ & $27.6 \%(8 / 29)$ \\
Local progression during chemotherapy & $6 / 78$ & $\mathrm{~N} / \mathrm{A}^{\mathrm{d}}$ & $21.1 \%(4 / 19)$ & $6.9 \%(2 / 29)$ \\
Pathological fractures & $2 / 78$ & $4.2 \%(2 / 48)$ & $0 \%(0 / 19)$ & $6.9 \%(2 / 29)$ \\
Nausea and vomiting & $60 / 78$ & $41.7 \%(20 / 48)$ & $52.6 \%(10 / 19)$ & $69.0 \%(20 / 29)$ \\
Leucopenia (combined with doxorubicin) & $56 / 78$ & $62.5 \%(30 / 48)$ & $47.4 \%(9 / 19)$ & $51.7 \%(15 / 29)$ \\
Increase in creatinine level & $5 / 78$ & $0 \%(0 / 48)$ & $5.3 \%(1 / 19)$ & $13.8 \%(4 / 29)$ \\
Increase in aspartate aminotransferase & $3 / 78$ & $0 \%(0 / 48)$ & $10.5 \%(2 / 19)$ & $3.5 \%(1 / 29)$ \\
Anemia & $4 / 78$ & $2.1 \%(1 / 48)$ & $10.5 \%(2 / 19)$ & $6.9 \%(2 / 29)$ \\
\hline
\end{tabular}

Notes: ${ }^{\mathrm{a}}$ Time interval for intra-arterial limb infusion of cisplatin was $3 \mathrm{hrs}$; ${ }^{\mathrm{b}}$ Time interval for intra-arterial limb infusion of cisplatin was 6 hrs. Abbreviations: CDDP, cisplatin; CTCAE, Common Terminology Criteria for Adverse Events; N/A, not available.

not prefer to increase the number of repetitions of IA infusion, as described by the other authors, ${ }^{3,4,9,14,15}$ but instead we preferred to transfer the patients directly to surgery in case of worse control of systemic disease. During our observation, we found that a sporadic case with the telangiectatic type might benefit more from local IA infusion of cisplatin than cases with other subtypes. Although there are diverse opinions on its chemosensitivity characteristic, in recent years, more and more studies have proved that this subtype is chemo-sensitive and has the same or even better long-term survival than the other subtypes. However from our experience, although no obvious distinction was noted in tumor response rates compared with the other subtypes of osteosarcoma, which might be partly due to the small sample size of this study, the telangiectatic osteosarcomas with greater tumor volume, which had larger blood-filled cavities, separated by septa containing highly malignant cells, with a more predominately lytic radiographic pattern, might show a local poorer response to systemic treatment. IA administration of cisplatin in these cases might be beneficial for adding intensive high-dose chemotherapeutic agents to high malignant tumor cells located in the pseudocapsule wall. Further studies with a larger sample size of this subtype and prospective trials should be designed to testify this hypothesis.

Limited complications following placement of an IA catheter for extremity infusion of chemotherapy have been reported in majority of studies. ${ }^{3,10,14,28}$ Cullen et al, ${ }^{14}$ performed 408 IA infusions of cisplatin in 109 patients with osteosarcoma, and only three minor complications were reported, including painful arterial spasm and local hematoma. However, in our study, a certain proportion of the population developed skin or muscle necrosis after IA infusion. After reviewing the arteriographies, we observed that the position of the catheter might influence the outcomes. We tended to place the catheter into the appropriate arterial bed supplying the neoplasm. If it was placed in the main trunk of the arteries, which also supplied some part of the skin or muscle, the probability of developing skin or muscle necrosis was elevated. Selective arteriography of the more distal vessels, such as the deep femoral artery, was performed when this information was needed to determine the total or partial contribution to the tumor supply. Besides, we just used an ordinary infusion pump to deliver the drug. Nishimura et al, ${ }^{30}$ chose to deliver cisplatin in a pulsatile jet fashion with the use of a Wallace-Gianturco pump in an attempt to achieve a more homogeneous distribution of drug throughout the tumor. Non-distribution of the drug to the related arteries might exacerbate vascular spasm and induce thrombus formation, which might also partly cause unexpected complications.

\section{Limitations}

There are several limitations to this study. Firstly and most important of all, too many questions have been raised and many cannot be satisfactorily answered by a retrospective study design especially with a relatively small number of patients from a single institution. Secondly, strict randomization of patients was not feasible. Selection bias was inevitable in patients with a larger tumor size or tumors adjacent to neurovascular bundles tended to receive IA infusion. However, we tried to achieve a balanced distribution of gender, 
age, tumor location, stage, and other clinical characteristics as much as possible to compare survival differences. Thirdly, there were no uniform chemotherapy protocols for all the patients, and the sequence, as well as combination of four agents used, was heterogeneous in either the IV or the IA group. We could only classify these different combinations into those with or without ifosfamide, or those with neoadjuvant chemotherapy for less than 8 weeks or longer than 8 weeks. However, we did not consider this point as a major weakness of the study because there was no observed difference in survival when various protocols based on the same four agents were used according to previous reports. ${ }^{1,31}$ Finally, surgical margins were occasionally difficult to distinguish because different surgeons operated on these patients during the five-year period. The surgical techniques used by the surgeons or by even one surgeon should be considered to have improved with time, which might also be a confounder when interpreting the result. However, both groups of patients experienced the same problem; and compared with the other study with a time span of more than 10 years, this difference was not so obvious. But being a study investigating an orphan disease in a single institution, this patient population should not be considered as a small cohort.

\section{Conclusion}

We found no survival advantage of chemotherapy using IA infusion to improve the local response or survival in osteosarcoma of the extremities. The addition of ifosfamide or increase in the number of neoadjuvant doses might improve the tumor local response but without any benefit on survival. Arteriography for TNV can be used to predict the tumor histologic response with high sensitivity and considerable accuracy. Telangiectatic osteosarcoma might benefit from IA infusion. Malposition of the catheter might severely increase the complication of skin or muscle necrosis.

\section{Acknowledgments}

We thank all the patients for cooperating in intra-arterial cisplatin infusion and accepting our follow-up interview. We also thank Dr. Kunkun Sun and Dr. Danhua Shen for reviewing the pathological slides of these osteosarcoma patients and Dr. Yuan Li for conducting clinical bone scan or PET/CT evaluation of our patients. This work was supported by Beijing Municipal Science \& Technology Commission (Fund No. was Z171100001017907). The role of the sponsor had no involvement in any of the stages from study design to submission of the paper for publication.

\section{Author contributions}

All authors contributed to data analysis, drafting or revising the article, gave final approval of the version to be published, and agree to be accountable for all aspects of the work.

\section{Disclosure}

The authors report no conflicts of interest in this work.

\section{References}

1. Smeland S, Bielack SS, Whelan J, et al. Survival and prognosis with osteosarcoma: outcomes in more than 2000 patients in the EURAMOS-1 (European and American osteosarcoma study) cohort. Eur J Cancer. 2019;109:36-50. doi:10.1016/j.ejca.2018.11.027

2. Picci P, Sangiorgi L, Rougraff BT, Neff JR, Casadei R, Campanacci M. Relationship of chemotherapy-induced necrosis and surgical margins to local recurrence in osteosarcoma. J Clin Oncol. 1994;12 (12):2699-2705. doi:10.1200/JCO.1994.12.7.1422

3. Bacci G, Ruggieri P, Picci P, et al. Intra-arterial versus intravenous cisplatinum (in addition to systemic adriamycin and high dose methotrexate) in the neoadjuvant treatment of osteosarcoma of the extremities. results of a randomized study. J Chemother. 1996;8(1):70-81.

4. Ferrari S, Mercuri M, Picci P, et al. Nonmetastatic osteosarcoma of the extremity: results of a neoadjuvant chemotherapy protocol (IOR/OS-3) with high-dose methotrexate, intraarterial or intravenous cisplatin, doxorubicin, and salvage chemotherapy based on histologic tumor response. Tumori. 1999;85(6):458-464. doi:10.1177/030089169908500607

5. Bacci G, Picci P, Ruggieri P, et al. Primary chemotherapy and delayed surgery (neoadjuvant chemotherapy) for osteosarcoma of the extremities. The istituto rizzoli experience in 127 patients treated preoperatively with intravenous methotrexate (high versus moderate doses) and intraarterial cisplatin. Cancer. 1990;65(11):2539-2553.

6. Winkler K, Bielack S, Delling G, et al. Effect of intraarterial versus intravenous cisplatin in addition to systemic doxorubicin, high-dose methotrexate, and ifosfamide on histologic tumor response in osteosarcoma (study COSS-86). Cancer. 1990;66(8):1703-1710. doi:10.1002/1097-0142(19900915)66:14+<1326::aidcncr2820661405>3.0.co;2-p

7. Jaffe N, Raymond AK, Ayala A, et al. Effect of cumulative courses of intraarterial cis-diamminedichloroplatin-II on the primary tumor in osteosarcoma. Cancer. 1989;63(1):63-67. doi:10.1002/1097-0142 (19890301)63:5<948::aid-cncr2820630527>3.0.co;2-u

8. Bacci G, Ferrari S, Tienghi A, et al. A comparison of methods of loco-regional chemotherapy combined with systemic chemotherapy as neo-adjuvant treatment of osteosarcoma of the extremity. Eur $J$ Surg Oncol. 2001;27(1):98-104. doi:10.1053/ejso.2000.1056

9. Avritscher R, Javadi S. Transcatheter intra-arterial limb infusion for extremity osteosarcoma: technical considerations and outcomes. Tech Vasc Interv Radiol. 2011;14(3):124-128. doi:10.1053/j.tvir.2011.02.004

10. Hugate RR, Wilkins RM, Kelly CM, Madsen W, Hinshaw I, Camozzi AB. Intraarterial chemotherapy for extremity osteosarcoma and MFH in adults. Clin Orthop Relat Res. 2008;466(6):1292-1301. doi:10.1007/ s11999-008-0396-z

11. Gill M, McCarthy M, Murrells T, Silcocks P. Chemotherapy for the primary treatment of osteosarcoma: population effectiveness over 20 years. Lancet. 1988;1(8587):689-692. doi:10.1016/S0140-6736(88) 91485-7

12. Fuchs N, Bielack SS, Epler D, et al. Long-term results of the cooperative German-Austrian-Swiss osteosarcoma study group's protocol COSS- 86 of intensive multidrug chemotherapy and surgery for osteosarcoma of the limbs. Ann Oncol. 1998;9(8):893-899. doi:10.1023/A:1008391103132 
13. Eisenhauer EA, Therasse P, Bogaerts J, et al. New response evaluation criteria in solid tumours: revised RECIST guideline (version 1.1). Eur J Cancer. 2009;45(2):228-247. doi:10.1016/j.ejca.2008.10.026

14. Cullen JW, Jamroz BA, Stevens SL, et al. The value of serial arteriography in osteosarcoma: delivery of chemotherapy, determination of therapy duration, and prediction of necrosis. J Vasc Interv Radiol. 2005;16(8):1107-1119. doi:10.1097/01.RVI.0000167856.31329.F8

15. Chiu TJ, Wang JW, Chen YJ, Tang Y, Huang CH. Intraarterial cisplatin and intravenous adriamycin in nonmetastatic osteosarcoma of the extremities: a single institution experience in Taiwan. Chang Gung Med J. 2009;32(1):72-80.

16. Brochtrup ED, Smee NM, Armbrust LJ, Caserto BG. Pathology in practice. Telangiectatic osteosarcoma. J Am Vet Med Assoc. 2013;242 (10):1355-1357. doi:10.2460/javma.242.5.611

17. Liu JJ, Liu S, Wang JG, et al. Telangiectatic osteosarcoma: a review of literature. Onco Targets Ther. 2013;6:593-602.

18. Angelini A, Mavrogenis AF, Trovarelli G, Ferrari S, Picci P, Ruggieri P. Telangiectatic osteosarcoma: a review of 87 cases. J Cancer Res Clin Oncol. 2016;142(10):2197-2207. doi:10.1007/s00432-016-2126-3

19. Durnali A, Alkis N, Cangur S, et al. Prognostic factors for teenage and adult patients with high-grade osteosarcoma: an analysis of 240 patients. Med Oncol. 2013;30(3):624. doi:10.1007/s12032-013-0624-6

20. Collins M, Wilhelm M, Conyers R, et al. Benefits and adverse events in younger versus older patients receiving neoadjuvant chemotherapy for osteosarcoma: findings from a meta-analysis. J Clin Oncol. 2013;31(18):2303-2312. doi:10.1200/JCO.2013.49.0219

21. Xu J, Xie L, Guo W. Neoadjuvant chemotherapy followed by delayed surgery: is it necessary for all patients with nonmetastatic high-grade pelvic osteosarcoma? Clin Orthop Relat Res. 2018;476(11):21772186. doi:10.1097/CORR.0000000000000387

22. Colvin MP, Curran JP, Jarvis D, O'Shea PJ. Femoral artery pressure monitoring. Use of the seldinger technique. Anaesthesia. 1977;32 (5):451-455. doi:10.1111/j.1365-2044.1977.tb10048.x

23. Picci P, Bacci G, Campanacci M, et al. Histologic evaluation of necrosis in osteosarcoma induced by chemotherapy. Regional mapping of viable and nonviable tumor. Cancer. 1985;56(7):1515-1521. doi:10.1002/ 1097-0142(19851001)56:7<1515:AID-CNCR2820560707>3.0.CO;2-6
24. Enneking WF, Spanier SS, Goodman MA. A system for the surgical staging of musculoskeletal sarcoma. Clin Orthop Relat Res. 1980;153:106-120.

25. Chung AE, Shoenbill K, Mitchell SA, et al. Patient free text reporting of symptomatic adverse events in cancer clinical research using the national cancer institute's Patient-Reported Outcomes version of the Common Terminology Criteria for Adverse Events (PRO-CTCAE). $J$ Am Med Inform Assoc. 2019;26(4):276-285. doi:10.1093/jamia/ocy169

26. Liu YJ, Zhu GP, Guan XY. Comparison of the NCI-CTCAE version 4.0 and version 3.0 in assessing chemoradiation-induced oral mucositis for locally advanced nasopharyngeal carcinoma. Oral Oncol. 2012;48(6):554-559. doi:10.1016/j.oraloncology.2012.01.004

27. Bacci G, Picci P, Ferrari S, et al. Primary chemotherapy and delayed surgery for nonmetastatic osteosarcoma of the extremities. Results in 164 patients preoperatively treated with high doses of methotrexate followed by cisplatin and doxorubicin. Cancer. 1993;72(11):32273238. doi:10.1002/1097-0142(19930901)72:5<1657::aid-cncr282072 $0527>3.0 . \operatorname{co} ; 2-n$

28. Jaffe N, Knapp J, Chuang VP, et al. Osteosarcoma: intra-arterial treatment of the primary tumor with cis-diammine-dichloroplatinum II (CDP). Angiographic, pathologic, and pharmacologic studies. Cancer. 1983;51(3):402-407. doi:10.1002/1097-0142(19830201) 51:3<402::AID-CNCR2820510308>3.0.CO;2-P

29. Stewart DJ, Benjamin RS, Zimmerman S, et al. Clinical pharmacology of intraarterial cis-diamminedichloroplatinum(II). Cancer Res. 1983;43(2):917-920.

30. Nishimura Y, Yoshimura H, Iwata K, et al. [Distribution of pulsed intra arterial infusion chemotherapy in hepatic carcinomas]. Gan To Kagaku Ryoho. 1989;16(8 Pt 2):2991-2994.

31. Carrle D, Bielack SS. Current strategies of chemotherapy in osteosarcoma. Int Orthop. 2006;30(6):445-451. doi:10.1007/s00264-006-0192-x

\section{Publish your work in this journal}

Cancer Management and Research is an international, peer-reviewed open access journal focusing on cancer research and the optimal use of preventative and integrated treatment interventions to achieve improved outcomes, enhanced survival and quality of life for the cancer patient
The manuscript management system is completely online and includes a very quick and fair peer-review system, which is all easy to use. Visit http://www.dovepress.com/testimonials.php to read real quotes from published authors. 